Nicola CARDIA

Università Comenius di Bratislava

\title{
IL NEOPURISMO E LA POLITICA LINGUISTICA DEL FASCISMO
}

Le antiche tendenze puristiche connesse con la questione della lingua, all'insegna della dicotomia «norma-uso », «lingua letteraria - lingua d'uso comune $»^{1}$, riemergono intorno agli anni Trenta del Novecento, riportate in auge dalla politica linguistica instaurata dal fascismo e ispirate ad un più generale orientamento nazionalista ed esterofobo.

I nuovi termini storici della questione della lingua vengono a coincidere con l'orientamento purista e protezionistico, cui si ispira la politica linguistica del fascismo, accompagnata da una martellante campagna di sensibilizzazione tramite gli organi di stampa ${ }^{2}$ e finalizzata a depurare la lingua italiana da un copioso numero di forestierismi. Il programma di bonifica linguistica adottato dal regime fascista prende avvio con l'introduzione di una tassa sulle insegne straniere l'11 febbraio 1923, culminante nel decreto legge del 18 gennaio 1939, che comminava pene severe ai locali pubblici che non avessero nomi stranieri. Quindi, nell'anno 1940, in un clima di crescente xenofobia e di caccia ai forestierismi, l'Accademia dei Lincei (allora Accademia d'Italia) nominava una commissione col compito di esaminare i singoli esotismi e di proporne l'accettazione, l'adattamento o la sostituzione ${ }^{3}$.

Fra i linguisti maggiormente accreditati, i quali intervennero direttamente o vennero consultati nell' ambito della campagna linguistica condotta dal fascismo contro gli esotismi, spicca per la sua autorevolezza la personalità di Bruno Migliorini, il quale introdusse con successo alcune sostituzioni (come ad esempio autista per chauffeur), mentre invece numerose sue altre proposte non vennero avallate (ad es. arlecchino in luogo di cocktail o direttoriale per editoriale).

Per ciò che concerne la principale fonte di provenienza degli esotismi durante il periodo fascista, il predominio quantitativo delle voci francesi risulta legato alla circostanza rilevata dal Migliorini, che «l'apertura verso l'estero era in quegli anni

\footnotetext{
${ }^{1}$ All'inizio del Novecento tali tendenze apparivano svuotate di ogni ragione di sussistenza, soffocate dalla reazione di un generale moto di stanchezza, coagulatasi in un'aperta ostilità antipurista, alimentata dal liberalismo linguistico professato dal Croce, (si veda il programma linguistico per le scuole elementari, approntato nel 1922 da G.Lombardo Radice, direttore dell'istruzione elementare). Degno di rilievo è il fatto che la restaurazione delle tendenze puristiche avvenisse poco dopo la soppressione dell'Accademia fiorentina della Crusca, voluta dal Croce e dal De Lollis e decretata da Gentile, ministro dell'istruzione nel primo Gabinetto fascista.

2 Apriva la crociata contro gli esotismi l'articolo apparso il 16 agosto 1926 sulla «Nuova Antologia », La difesa della lingua italiana, nel quale l'articolista, membro del pertito fascista, dopo avere deprecato l'uso della parola forgiare o di termini come messa a punto, ruolo, esortava i camerati (un altro francesismo) ad un'intensa azione di bonifica linguistica. A quest'articolo ne seguirono altri di identico tenore, apparsi ancora nella « Nuova Antologia» (Per la difesa della lingua italiana, 1. novembre 1926; Fuori i barbari, 1 marzo 1928; Barbaro dominio!, Milano, 1933)

${ }^{3}$ Fra gli esotismi sottoposti al vaglio e poi promossi: sport, tennis, picnic, bar. Fra quelli invece bocciati: festival (che bisognava sostituire con festivale), parquet (con parchetto), gin (gineprella), cognac o brandy (con la parola ratafià), cocktail (con arlecchino), hotel (con albergo), garage (con rimessa). Un vocabolo destinato a sollevare accese discussioni fu la parola bar, i cui equivalenti nazionali avrebbero dovuto essere a seconda delle opinioni bettolino, quisibeve, taberna potoria, ber, barro, barra, bara, mescita, liquoreria, taverna
} 
limitata per lo più alla conoscenza del francese. Molto più ristretta è in confronto la conoscenza del tedesco e dell'inglese ${ }^{4}$. Una testimonianza, questa, che, rapportata alla situazione di oggi, fa risaltare tutta la distanza che separa la dinamica della penetrazione degli esotismi durante quegli anni da quella di oggi. Questo, se si considera la massiccia affluenza di anglicismi nelle lingue nazionali veicolati dalla civiltà tecnologica, difficilmente contrastabile con rigidi quanto anacronistici provvedimenti di protezionismo linguistico attuati dallo Stato (si veda la Francia a partire dagli anni Settanta del secolo scorso). Sarebbe d'altra parte impossibile sottovalutare il particolare spessore di risonananza universale e di schiacciante supremazia dell'anglo-americano che incide sensibilmente sulla dinamica di evoluzione dell'italiano contemporaneo, coinvolgendo tutti i campi, dallo sport alla politica, dall'economia all'informatica.

Tornando alle nostre riflessioni sulla politica linguistica attuata dal fascismo durante il Ventennio (1923-1943), riteniamo opportuno rilevare la circostanza che l'intransigente rigorismo purista, ispirato ad una politica di autarchia linguistica e diretto principalmente ad eliminare i francesismi presenti nella lingua italiana, trova riscontro nell'elaborazione di una cospicua produzione normativa (leggi, circolari, decreti) e di una capillare propaganda intimidatoria che coinvolse la scuola, la radio e la stampa ${ }^{5}$. Da qui la compilazione di dettagliati elenchi di vocaboli proscritti, sottoposti a veto ${ }^{6}$ e destinati ad essere opportunamente modificati.

La politica linguistica del fascismo ci pare riassumibile in tre momenti principali, riconducibili : a)ad un obiettivo di unificazione linguistica attraverso un'operazione capillare di dialettofobia attuata principalmente tramite la scuola ; b) ad una lotta senza quartiere, mirata a soffocare le lingue delle minoranze linguistiche ; c) ad una restaurazione intransigente degli ideali di purismo linguistico, diretta a rinnovare la purezza nazionale della lingua, inquinata dalla presenza di esotismi ${ }^{7}$.

\footnotetext{
${ }^{4}$ Sugli esotismi presenti nella lingua italiana così il Migliorini : «Negli ultimi anni si è reagito a questa invasione con spirito fascista, e così un gran numero d'intrusi sono stati eliminati o almeno assimilati. Così invece di record si dice primato; non si dice più regisseur ma regista. Nelle trattorie e negli alberghi i menus si chiamano liste, e nessuno si vergogna a chiamare bambinaia quella che si chiamava bonne. Il Touring Club italiano ha cambiato il proprio nome in Consociazione turistica italiana. Il Duce ha dato l'esempio, quando, visitando nel 1931 una mostra d'arte che si stava per inaugurare ha chiamato vernice, quella che prima si indicava con il vocabolo francese vernissage (cioè la verniciatura dei quadri, che una volta gli artisti facevano alla vigilia dell'inaugurazione, in presenza di invitati) ». (MIGLIORINI, $1941: 410$ )

${ }^{5}$ Sulle prese di posizione contro l'esterofobia linguistica indiscriminata così il De Mauro : «nel 1941 (e la data va sottolineata) A.Menarini, a proposito di bar-barista scriveva : «...Parole come bar e film non ci sono state imposte ed hanno raggiunto tanta popolarità unicamente per opera nostra; insorgendo rabbiosamente contro di esse solo perchè straniere o perchè ci provengono da un Paese ora nemico, non paleseremmo la coscienza della nostra superiorità... dopo quelle inglesi, uguale trattamento avrebbero dovuto richiedere le voci greche..., ma una simile impresa non renderebbe un buon servigio alla nostra lingua, che pur deve parte della sua duttilità, del suo colore, della sua espressività e specialmente della sua ricchezza al materiale d'importazione ». (MeNARINI, 1941 : 113-118)

${ }^{6}$ Vedi ad es.la rubrica curata da Paolo Monelli sulla «Gazzetta del popolo » nel 1932-1933, intitolata Una parola al giorno, oppure il programma radiofonico La lingua d'Italia, prodotto nel 1938 dall'EIAR, con l'Accademia d'Italia

${ }^{7}$ Il colore bordeaux divenne «color barolo »; il tessuto principe di Galles fu semplicemente il «tessuto principe »; per film venne adottata la parola «pellicola»; per apache «teppista»; per
} -44 - 
Per quanto concerne il progressivo regresso dei dialetti occorre dire che, assai più della battaglia ad oltranza condotta dal fascismo contro i dialetti in nome della purezza e dell'unità della lingua nazionale, si sarebbe rivelata assai più incisiva per il declino dei dialetti l'azione svolta dall'urbanesimo e dalla conseguente situazione di osmosi linguistica. Un'azione di progressiva erosione questa, che coinvolse non solo gli immigrati ma pure la popolazione locale residente nella località di destinazione degli emigrati.

A tale riguardo occorre tuttavia sottolineare che il processo di osmosi linguistica e di italianizzazione dei dialetti (sia delle strutture lessicali, che di quelle fonologiche, morfologiche e sintattiche) connessa con l'urbanesimo non si è limitato soltanto ai maggiori centri urbani. In realtà, come osserva il De Mauro, esso si è esteso «pure ai centri minori raggiunti dalle migrazioni stagionali, che favoriscono i prestiti interdialettali e portano talora alla formazione di idiomi nascenti dal compromesso fra o più sistemi linguistici dialettali, il cui uso è spesso riservato agli individui appartenenti alle categorie più direttamente impegnate nel fenomeno migratorio » (DE MAURO, 1970 : 72 - 73).

Di particolare interesse risulta il fatto che già nel 1930 il linguista M.Bartoli, membro dell'Accademia d'Italia, mostrasse di avere una chiara percezione del progressivo indebolimento dei dialetti, quando notava : «Oggi nei grandi centri industriali di Torino e Milano, come negli emporii commerciali di Genova e Trieste affluiscono italiani di ogni regione... portati naturalmente a usare, piuttosto che i dialetti la lingua nazionale italiana... Sicchè le città, che ieri furono le culle delle varietà dialettali, saranno domani le loro tombe »(BARTOLI, $1930: 68$ ).

Al di là del prevalente giudizio negativo sulla sostanza della politica linguistica nazionalistica ${ }^{8}$ e xenofoba instaurata dal fascismo, ci pare innegabile che essa venga a coincidere con una fase storica assai significativa della questione della lingua nel primo Novecento. Una fase storica, questa, che si presenta sotto il segno di un purismo deteriore e retrivo, così come risulta da tante dispute linguistiche intorno ad alcuni esostismi e a cui non rimase estraneo il mondo culturale e scientifico, sceso in campo attraverso numerosi interventi di fiancheggiamento. Fra i suoi principali promotori insigni letterati come Alfredo Panzini, ma soprattutto autorevoli linguisti come Bruno Migliorini, Alfredo Schiaffini e Giulio Bertoni, esponenti della corrente del neopurismo.

Un'analisi a posteriori di carattere sociolinguistico della politica linguistica attuata con notevole tenacia ma con scarsa coerenza dal regime fascista, implica a nostro avviso una serie di riflessioni di carattere preliminare e di definizioni terminologiche, utili a tracciare un quadro teorico-concettuale, entro cui collocare la questione della politica linguistica (language policy / politique linguistique /Sprachen Politik / jazyková politika), concetto questo correlativo alla nozione di

\footnotetext{
claxon «tromba o sirena »; «carovana » venne preferita a roulotte. Infine, termini italianissimi come «insalata russa o chiave inglese » venivano messi al bando, in quanto evocatori di nazioni nemiche, sostituiti dai più patriottici « insalata tricolore »e « chiavemorsa ».

${ }^{8}$ La studiosa altoatesina G. Klein, esaminando la politica linguistica del fascismo sotto un profilo sociolinguistico, osserva : «il dibattito su questi problemi di politica linguistica ha come retroterra ideologico la vecchia questione della lingua con le sue convinzioni puristiche e nazionalistiche basate sull'equiparazione (storicamente non provata) fra lingua e nazione, fra lingua e popolo » (KLEIN, $1986: 22)$.
} 
pianificazione linguistica (language planning / planification linguistique / Sprachplanung).

Nel caso specifico, saremmi inclini ad adottare, d'accordo con la Klein, il termine più generale e onnicomprensivo di «politica linguistica ${ }^{9}$ piuttosto che quello assai più specifico di «pianificazione linguistica», che implica un' accentuata idea di intenzionalità. Questo a causa dell'assenza di un programma di pianificazione linguistica, coerente e sistematica ${ }^{10}$ che tenesse sufficientemente conto della diversità ed eterogeneità dei codici linguistici all'interno del territorio nazionale, vale a dire di situazioni di diglossia e/o situazioni di bilinguismo sociale all'interno di una comunità linguistica.

Sarebbe difficile negare, tuttavia, la presenza di alcuni elementi inerenti a un programma di pianificazione linguistica, fra cui soprattutto la codificazione normativa, che, nel caso appunto del fascismo, si serve allo scopo di una standardizzazione forzata delle norme d'uso di modalità formali, severamente monocentriche e endonormative. Una standardizzazione, questa, attuata attraverso misure d'intervento e strumenti normativi cogenti (leggi prescrittive e proscrittive) che vengono a coinvolgere precise aree d'intervento; in primo piano le comunicazioni di massa in generale, e, più in particolare. la stampa, la radio, il cinema (cfr. FISHMAN, 1972).

Ricapitolando, gli aspetti più peculiari e distintivi della questione della lingua, riportata in vita dal fascismo e strumentalmente adattata e subordinata alle sue particolari esigenze ideologiche, tipiche di un regime totalitario, vorremmo sottolineare tre componenti principali, che animano la maggior parte dei dibattiti linguistici negli anni Trenta.

Il primo è l'aperta ostilità nei confronti del dialetto e di ogni forma di regionalismo linguistico, inteso come elemento disgregante dell'unità della lingua ; il secondo l'ostilità verso le lingue delle minoranze ; il terzo infine, come si è già detto, è orientato contro la presenza di vocaboli stranieri (soprattutto francesi) provenienti dalle nazioni nemiche.

Il denominatore comune che amalgama queste tre componenti ci sembra riconducibile al criterio nazionale della difesa ad oltranza della lingua nazionale e dell'unità della lingua ${ }^{11}$, associata a quella correlativa della sua purezza. Da qui l'aperto misoneismo e la chiara matrice reazionaria alla quale appare ispirarsi tale politica - assai pertinente ci pare la formula « giacobinismo linguistico » (Renzi) -

\footnotetext{
${ }^{9}$ All'interno della definizione della norma linguistica, che presuppone un esame preliminare del repertorio linguistico di una comunità linguistica, alle note categorie funzionali, applicabili per una sua suddivisione e basate su Fishman (1971) e Hymes (1971) e rielaborate da Bell nel 1978, che riflettono gli statuti di lingua all'interno di una comunità (unica lingua ufficiale; lingua ufficiale aggiuntiva; lingua ufficiale regionale; lingua promossa; lingua tollerata; lingua scoraggiata) aggiungeremmo quella suggerita dalla Klein di lingua vietata.

${ }_{10}$ Gli strumenti per un'analisi approfondita delle politiche linguistiche e delle pianificazioni linguistiche vengono forniti, allo stato attuale della ricerca linguistica, dalla sociolinguistica e, in particolare, dalla sociolinguistica politica. La politica linguistica così, quale terreno specifico di studio nell'ambito della sociolinguistica, rappresentrebbe addirittura, almeno secondo Fishman (1972), il campo centrale della sociolinguistica applicata.

${ }^{11}$ In contrasto con il coro generale di consensi del mondo accademico ufficiale alla politica linguistica del fascismo, uno dei pochissimi ad attribuire un forte risalto alle varietà dialettali e regionali (vedi a riguardo G.G.Ascoli), affermando l'insussistenza del concetto di unità linguistica, fu nel 1939 Giacomo Devoto, proprio alla vigilia dello scoppio del secondo conflitto bellico.
} 
che si esplicano, come osserva la Klein «sotto forma di repressione delle varietà dei dialetti, delle lingue minoritarie e delle espressioni straniere ». Questo allo scopo di raggiungere il consenso nella ricerca di una unificazione linguistica che all'epoca non si era ancora realizzata nonostante gli interventi linguisticopianificatori fin dai primi anni dell'Unità d'Italia e «nonostante che l'ormai diffusa convinzione sull'utilità della dialettofonia come strumento nazionale di comunicazione si fosse radicata nella coscienza culturale media, convinzione tuttavia totalmente priva di effetti pratici ». (KLEIN, $1986: 23$ )

Gli ambiti principali, raggiunti dall'offensiva linguistica promossa dal fascismo (soprattutto dopo gli anni Trenta) che faceva leva sull'ideale della purezza della lingua e dell'unità della lingua, sono quelli della legislazione, dell'istruzione scolastica, come pure delle minoranze etniche linguistiche.

Tutto questo nell'imponente sforzo di affermare la validità del teorema autarchico e xenofobo, basato sulla strumentale equiparazione « una nazione = una lingua ».

Oltre alla purezza della lingua va sottolineata un'altra connotazione principale, ravvisabile nella politica linguistica puristica perseguita dal fascismo nel Ventennio e abilmente utilizzata come mezzo di propaganda del regime. Alludiamo qui all'impegno profuso da parte del partito fascista nel condurre una lotta senza quartiere contro la piaga dell'analfabetismo, allo scopo - come sottolinea un testimone dell'epoca Gianni Cugini nel 1933 - «di diffondere col ritmo accelerato fascista l'istruzione primaria fino ai più sperduti casolari delle terre nostre ».

Ancora a proposito della politica scolastica attuata dal fascismo va detto che esso considerava l'analfabetismo come una vera vergogna, sopprimendo dai censimenti ogni inchiesta su di esso e servendosene come efficace strumento di propaganda politica. Occorre inoltre aggiungere che tale politica assumeva il significato di una battaglia vuoi contro l'analfabetismo vuoi contro l'uso del dialetto (condotta sia attraverso l'azione della scuola che attraverso quella della stampa), in difesa del prestigio nazionale all'estero. Non è un caso che l'avversione esasperata verso il dialetto manifestata dal fascismo, che impronta i programmi scolastici italiani durante il Ventennio, trovi riscontro nelle istruzioni che il Ministero dell'Educazione in periodo fascista impartiva alla stampa ${ }^{12}$.

Sotto quest'aspetto sarebbe difficile negare che una delle iniziative mirate ad incentivare e accelerare il processo di alfabetizzazione della nazione (nella quale non mancano numerosi elementi validi e positivi) fosse rappresentata dalla riforma della scuola Gentile del $1923^{13}$, che introduceva il principio della gratuità, portando l'obbligo scolastico ai 14 anni di età.

\footnotetext{
${ }^{12}$ Si riportano qui alcuni stralci di circolari ministeriali inviate agli organi di stampa dal Ministero dell'Educazione, dove si legge : 25 luglio 1938: «Non occuparsi dell'antologia dei poeti milanesi contemporanei, a cura di S..Pagani... »; 22 settembre 1941 : «I quotidiani, i periodici e le riviste non devono più occuparsi del dialetto »; 2 settembre 1942 : «non occuparsi del teatro vernacolo. Questa disposizione ha carattere tassativo e permanente... »; giugno 1943 : «non occuparsi di produzioni dialettali e di dialetti in Italia, sopravvivenze del passato che la dottrina morale e politica del fascismo tende decisamente a superare ». (DE MAURO, $1970: 357$ )

${ }_{13}$ Assumono un forte risalto i numerosi elementi liberali e democratici di ispirazione crociana, presenti nella riforma dell'istruzione scolastica varata nel 1923 da G.Gentile. Leggiamo nei programmi della riforma, redatti da G.Lombardo Radice (che prevedevano fra l'altro esercizi di 
Strettamente connessa con il programma di scolarizzazione e di alfabetizzazione forzata si presenta una seconda importante direttiva di marcia, utile ad incanalare la strategia di pianificazione e la politica linguistica del fascismo ma priva di alcuni requisiti teorici di base imprescindibili per ogni intervento di moderna pianificazione linguistica. Primo fra tutti un necessario esame preliminare delle varietà linguistiche presenti. Il principio ispiratore di tale direttiva risulta essere un «purismo ideologico di stato », definibile con il termine mediato dalla sociolinguistica cultivation approach.

Il segno più distintivo di tale politica linguistica si identifica a nostro avviso nella natura retriva e deteriore di un purismo di stato, con accentuate connotazioni ideologiche, finalizzate allo scopo di depurare drasticamente la lingua dagli elementi e influenze inquinanti e di disturbo, dialettali e specialmente straniere. Ciò che occorre soprattutto rilevare è che tale politica linguistica xenofoba e misoneista veniva condotta con il consenso diretto o indiretto dei neopuristi ${ }^{14}$, all'insegna di una crescente intolleranza verso tutto ciò che era straniero o « estraneo » oppure che era in contrasto col proclamato principio di unità linguistica della nazione. Una politica, questa, destinata a radicalizzarsi nella seconda metà degli anni Trenta, nonostante alcune avvisaglie già nel 1923 con l'introduzione della tassa sulle insegne straniere.

Il principale terreno di attuazione della politica di bonifica linguistica perseguita da parte del fascismo, che si avvale del costante supporto a livello teorico del neopurismo e fa leva su rigidi meccanismi di controllo e di censura con caratteri marcatamente prescrittivi, è rappresentato dalla lotta indiscriminata contro i forestierismi. Essa viene condotta attraverso espliciti divieti normativi; dapprima nel 1930, nei confronti del cinema straniero, e quindi nell'anno 1934 contro l'impiego di vocaboli stranieri nei giornali. Divieti questi, va ricordato, incoraggiati per lo più dagli stessi neopuristi e destinati sempre più ad inasprirsi dopo il 1935, fino al divieto dell'uso di parole straniere nelle intestazioni delle ditte e nelle varie forme pubblicitarie.

Il tratto più distintivo di questo «purismo ideologico di stato » (Raffaelli) va ravvisato nel fatto che, a differenza del purismo classico di vecchio stampo che insorgeva contro i neologismi inerenti alla lingua letteraria, il purismo di stato introdotto dal fascimo si concentra pragmaticamente sui neologismi e gli esotismi, penetrati in particolare sulla lingua di uso comune e sulle lingue speciali.

Va precisato però che raramente gli effetti di tale politica linguistica toccavano direttamente il parlante della comunità linguistica, almeno nella quotidianità d'uso

traduzione dal dialetto) : «I programmi che seguono sono delineati in guisa da fare, per se stessi, obbligo al maestro di rinnovare continuamente la propria cultura attingendo non a manualetti, in cui si raccolgono le briciole del sapere, ma alle vive fonti della cultura del popolo. Queste fonti sono la tradizione popolare, così come essa vive perenne educatrice nel popolo..., e la grande letteratura... ». (LOMBARDO RADICE, $1925:$ 551-560)

${ }^{14}$ Bruno Migliorini, il principale teorico del nepurismo, ne definisce così la sostanza : «il carattere essenziale del purismo è la lotta contro ogni specie di innovazione. Il neopurismo, distinguendo tra forestierismi e neologismi, vuole saggiare gli uni e gli altri alla luce della linguistica strutturale e funzionale... D'altra parte il purismo tradizionale teneva d'occhio esclusivamente l'Italia ; il neopurismo vuole servire alle necessità italiane, ma reputerebbe cattiva politica chiudere gli occhi alla realtà europea. Il purismo aveva di mira soprattutto la lingua letteraria ; il neopurismo estende lo sguardo anche alle lingue speciali ». (MIGLIORINI, $1941: 47$ ) 
della lingua. Questo, se si esclude la controversia linguistica intorno all'uso del Voi imposto dal regime, col conseguente divieto di utilizzare il Lei, allora considerato erroneamente un ispanismo, ricalcato sulla forma di Usted $^{15}$.

Ad un orientamento ideologico normativo di matrice neopuristica si collega, in direzione di una reale unità della lingua (più presunta che reale), una serie di interventi rigidamente regolatori e normativi, destinati tuttavia a non produrre quegli effetti duraturi, prerogativa indispensabile per il successo di ogni intervento di politica linguistica normativa e di pianificazione a lungo termine.

L'imponente sforzo di italianizzazione puristica della lingua esercitato dal regime fascista culmina com'è noto nel 1940 nell'istituzione di un autorevole organo normativo e correttivo di controllo non a caso proprio in seno all' Accademia d'Italia, la più prestigiosa e rappresentativa istituzione culturale del regime, la Commissione per «l'italianità della lingua». A tale organo viene affidato l'incarico di fare piazza pulita di tutti gli esotismi allo scopo di assicurasi il controllo burocratico della politica linguistica, rendendo quasi assoluto il controllo ideologico dell'intera vita culturale della nazione e lo stesso ruolo egemonico del partito fascista allora al potere.

Come è noto, il compito della Commissione istituita dall' Accademia d'Italia (i cui poteri dopo il 1942 sarebbero stati ridotti, tanto che le sostituzioni proposte avrebbero assunto il carattere di semplice suggerimento) consisteva nel compilare liste di parole straniere, stabilendo, secondo criteri di necessità e di urgenza, quali di esse andassero sostituite con termini italiani, quali semplicemente italianizzate, quali tollerate o date già per acquisite nella lingua italiana.

Nell'ambito dell'intensa attività di teorizzazione e di codificazione esercitata dai rappresentanti del neopurismo (con in testa il Migliorini) uno dei temi centrali dei dibattiti linguistici si identifica con il tentativo di pervenire ad una norma ideale, fruibile dalla massa degli utenti linguistici. Questo, nonostante alla fine finisse per prevalere nel Migliorini l'idea che il soggetto depositario della norma linguistica legata al cosiddetto «buon gusto » rimanga un'élite culturale ed intellettuale. Da qui l'implicita esigenza, delegata in misura prioritaria agli organi ed istituzioni di stato, di contribuire attivamente a coltivare tale ideale di norma legata al buon gusto, attraverso una sistematica attività di formazione della coscienza linguistica, finalizzata all'individuazione della norma ideale legata al buon gusto. Lo strumento privilegiato era quello delle censure linguistiche e di coercizioni, di cui l'insegnamento scolastico avrebbe dovuto essere ovviamente il terreno di applicazione.

Il crescente sforzo esercitato dai neopuristi, che operavano a partire dal 1940 in seno alla Commissione dell'Accademia d'Italia, era quindi diretto da un lato all'individuazione di una norma linguistica ideale e stabile, dall'altro a definire i criteri regolatori per una eventuale eliminazione o accettazione degli esotismi

\footnotetext{
${ }^{15}$ La battaglia del regime per l'introduzione del Voi, che vide scendere in campo una nutrita schiera di fautori del Voi (dal giornalista Oreste del Buono, al letterato fiorentino Bruno Cicognani) porta nel 1938 al decreto di divieto firmato dallo stesso Mussolini di usare il Lei esteso a tutti gli impiegati dello stato (vedi a questo proposito anche il film del 1977 Una giornata particolare del regista Ettore Scola), fino all'istituzione di una Commissione per l'italianità della lingua presso l'Accademia d'Italia, della quale fu il principale patrocinatore Bruno Migliorini, teorico del neopurismo linguistico.
} 
« sia per quanto riguarda i criteri di adattamento (fonetico e/morfologico), di traduzione o per l'impiego di espressioni alternative ad hoc» (Schiaffini).

Il Migliorini ${ }^{16}$, ispirandosi a meccanismi di valutazione puristici, nel quale assume un'importanza preminente l'elemento di verifica della latinità di un vocabolo, fa frequente ricorso ad uno strumento metodologico, da lui definito « glottotecnica » cioè l'applicazione sistematica degli insegnamenti forniti dalla linguistica per la creazione dei singoli termini o per la revisione di nomenclature allo scopo di ottenere, « il massimo dei vantaggi e il minimo degli inconvenienti ».

Va precisato a questo punto che, al di là delle dichiarazioni programmatiche $\mathrm{e}$ della eterogeneità delle posizioni all'interno dello schieramento neopurista (dai più progressisti Bertoni e Ugolini, i quali teorizzavano da parte loro un asse linguistico ideale Roma-Firenze ${ }^{17}$ alla linea più conservatrice, rappresentata da Lupi, Martelli e Milani) sarebbe prevalsa alla fine un'impostazione di carattere monolitico, fondata sull'equazione lingua= nazione. Il risultato finale sarebbe stato una lotta senza quartiere diretta contro i dialetti attraverso severe censure e misure coercitive da parte delle istituzioni scolastiche durante il Ventennio.

Tracciando un rapido bilancio sull'intensa attività svolta dai linguisti neopuristi, legati in varia misura attraverso un rapporto di solidarietà e di consenso all'ideologia totalitaria del regime fascista, va sottolineato il consistente impegno profuso da parte di una consistente schiera di intellettuali e autorevoli linguisti. Essi, con un approccio rigidamente puristico e autarchico, conferivano all'antica questione della lingua il carattere di una intensa attività di bonifica linguistica. Un'attività, questa, orientata in direzione unitaria e caparbiamente esterofoba e basata da un lato sulla necessità normativa di avviare un'assidua attività regolatrice e di controllo della società, dall'altro lato invece su quella legislativa, finalizzata ad una difesa protezionistica della purezza e unità della lingua nazionale.

Lo scopo principale di tale campagna di stato era, come si è detto, quello di ripristinare la purezza della lingua, liberandola da tutti gli elementi estranei, inquinanti o di disturbo, vale a dire gli esotismi linguistici e i dialettalismi.

L'aspetto forse più sconcertante, sul quale vorremmo attirare l'attenzione, connesso con l'intensa attività di bonifica linguistica esercitata dei neolinguisti, ci pare quello legato ad una visione di fondo piuttosto angusta, provinciale e statica delle questioni linguistiche. Si trattava nella sostanza di dispute accademiche, che vedevano impegnati alcuni fra i più autorevoli linguisti di quegli anni, del tutto scisse però dal grande fermento di riflessioni linguistiche che accompagnavano i progressi della scienza linguistica in Europa, dalla Scuola di Praga a quella di Copenaghen.

\footnotetext{
${ }^{16}$ La preoccupazione normativa accompagnò costantemente il Migliorini anche dopo la guerra ai livelli più diversi (dal Dizionario di ortografia e di Pronuncia, apparso a Roma nel 1969, alla collaborazione col «Corriere della Sera », durata fino al 1974). Va detto però che, sebbene fosse il teorico di punta del neopurismo, si sottrasse al rischio di contaminazioni della questione della lingua con quella della razza come pure a più plateali manifestazioni di connivenza con il regime fascista.

17 Va qui precisato che per «lingua di Roma» non si intendeva il dialetto romanesco, quanto piuttosto la lingua colta romana, come quella riportata nel Prontuario di pronunzia e ortografia, elaborato nel 1939 da G. Bertoni e F. Ugolini.
} 
Uno degli aspetti più significativi che ci preme sottolineare è il fatto che la politica linguistica attuata dal fascismo nell'arco di tempo compreso fra il 1932 e il 1940 non era sostenuta da un'indispensabile attività di pianificazione a lungo termine, necessario presupposto per il successo di ogni politica linguistica.

Per quanto riguarda le peculiarità teorico-metodologiche che caratterizzano la politica linguistica perseguita dal fascismo, (i cui tratti più distintivi e peculiari sono quelli dell'autarchia e del purismo o di stato), una delle sue maggiori peculiarità, legate alla definizione della norma linguistica è a nostro giudizio la drastica riduzione strumentale delle varietà linguistiche, presenti entro il repertorio della comunità linguistica nazionale. Varietà queste che nel caso del fascismo risultano sottomesse all'ideale monolitico di un italiano unitario e standardizzato (una standardizzazione quindi monocentrica) col conseguente riconoscimento di uno «status » nettamente subordinato sia ai dialetti che alle lingue delle minoranze etniche, cui viene attribuito, nell'ambito dei vari status da noi abbozzati, lo status subalterno di lingua «scoraggiata ».

Per ciò che concerne l'ottica di pianificazione linguistica perseguita dal regime fascista, ci pare essa abbracci una latitudine dinamicamente assai ampia, partendo da un'ottica di base definibile micro e intralinguistica (lotta contro i regionalismi ed esotismi) per dilatarsi via via ad un livello di ottica macro e extralinguistica (disposizioni coercitive contro i dialetti, i gerghi e le lingue minoritarie).

Passando ad esaminare le caratteristiche di approccio, vorremmo attirare l'attenzione sulla presenza nella politica linguistica del fascismo di alcuni tratti distintivi di un cultivation approach, considerata l'accentuata peculiarità di purismo linguistico, associata ad un'estrema preoccupazione regolativa e legislativa. Accanto al tratto caratteristico di cultivation approach ci pare ravvisabile anche la componente di policy approach, "mirando essa da un lato alla stabilità del codice linguistico prescelto (codificazione di una lingua ufficiale) dell'italiano standardizzato nei confronti dei dialettofoni e dall'altro all'estensione funzionale della lingua ufficiale (differenziazione funzionale per quanto riguarda i gruppi minoritari ». (KLEIN, $1986: 44)$

Per quanto riguarda i datori della norma, essi ci sembrano individuabili ad un duplice livello : decisionale (Ministero dell'Interno fascista) e nello stesso tempo anche esecutivo, (l'Accademia d'Italia), essendo i datori della norma presenti contemporaneamente sia all'interno del governo sia all'esterno di esso. Ciò trova riscontro nella costante azione di fiancheggiamento e di aperto sostegno alle direttive ideologiche del regime, che vede coinvolti in varia misura linguisti come Schiaffini, Migliorini, Bertoni, ecc.

Quanto alle motivazioni di fondo, implicite in tale politica linguistica, esse risultano essere prevalentemente di controllo sociale, fondendo il proclamato ideale fascista di unità politica nazionale con quello di un'ideale unità linguistica, in conformità con un orientamento ispirato ad un «purismo ideologico di stato » (Raffaelli, al quale appare associabile quella di «giacobinismo linguistico» (Renzi). Formule queste entrambe utili ad attirare l'attenzione sulle connotazioni marcatamente ideologiche, riconducibili all'autarchia e al protezionismo linguistico, conformemente all'ideologia e alla politica economica e commerciale professata dal regime fascista.

Ben più arduo si presenta il compito di analizzare la complessa rete di rapporti, a volte contraddittori, di solidarietà e collaborazione, ma anche di diffidenza fra 
l'entourage culturale (più precisamente i rappresentanti più autorevoli della scienza linguistica) e il regime fascista.

Questo a causa dell'obiettiva difficoltà di valutare la misura di questo consenso e l'eventuale peso preponderante di una delle due forze sinergiche in gioco (il regime da una parte e l'attività di consapevole fiancheggiamento dei linguisti dall'altra), nonchè a causa del complesso intreccio di rapporti e di reciproche convergenze. Ci pare lecito tuttavia ipotizzare l'esistenza di numerosi elementi di consonanza fra gli indirizzi culturali teorici e le forti predisposizioni di un regime totalitario (non solo quello fascista) ad incoraggiare e convogliare tendenze linguistiche autarchiche.

La legittimità della tesi prevalente fra gli storici della lingua, da noi condivisa, circa l'esistenza di una sostanziale identità di orientamento e di un complesso intreccio tra cultura e potere politico, trova un utile riscontro nell'articolo apparso nel 1937 su un giornale del tempo dal titolo «La lingua e la terra etiopica». Appare qui evidente il riferimento alla proposta autarchica di Bertoni-Ugolini, con l'implicita intenzione di espandere la lingua italiana fuori d'Italia, in particolare nelle colonie d'Africa.

Altrettanto arduo risulta il compito di dare una risposta univoca alla questione tuttora aperta di valutare la misura del successo della politica linguistica attuata durante il fascismo, essendo essa legata agli interessi del regime politico al potere. Sotto quest'aspetto ci pare che trovi conferma la fondatezza del principio di base che un successo duraturo di ogni politica linguistica impostata a lungo termine vada rapportato sia all'efficacia degli interventi di acculturazione, sia alla stessa capacità di incidere in profondità sui modelli di comportamento linguistico della comunità, sottoposta all'azione di una strategia persuasiva, che fa leva sul ricorso a canali quali la scuola o i mass media.

Nel caso del fascismo riteniamo di potere affermare che esso fallisca in definitiva nel suo tentativo di sradicare quei fattori considerati disgreganti, identificati appunto nei dialetti e nelle lingue delle varie minoranze etniche, destinate a riemergere in vario modo dopo la caduta del regime. Per altro verso, tuttavia, sarebbe difficile negare che il fascismo abbia contribuito a consolidare una diffusa tendenza normativa, atta a favorire un processo centripeto in direzione della standardizzazione linguistica (monocentrica), promossa dalla classe egemonica al potere, a svantaggio delle classi socialmente più indigenti ed emarginate.

Spostandoci all'interno di una prospettiva a lungo termine riteniamo tuttora aperta la domanda relativa alle ripercussioni a livello di comportamento linguistico, determinate dalla politica linguistica del fascismo ispirata alla xenofobia e alla lotta contro gli esotismi e attuata attraverso una serie di disposizioni legislative coercitive.

La principale difficoltà è legata a nostro giudizio al fatto che il materiale linguistico a disposizione si riferisce per lo più al registro scritto della lingua e legato a settori, sottoposti, per la loro stessa natura, ad un notevole controllo governativo, mentre rimane escluso il registro parlato della lingua lingua, legato all'uso quotidiano. A tale proposito occorrerà soprattutto tener conto, nell' ambito di un'analisi delle espressioni sottoposte ad interventi puristici regolatori e correttivi, del carattere effimero e contingente di non pochi adattamenti. 
Prima di concludere le nostre riflessioni, vorremmo ribadire il principio, valido specialmente nel caso della politica linguistica attuata dal fascismo, che ogni politica linguistica nei suoi effetti a livello di comportamento linguistico vada necessariamente inquadrata in un più ampio contesto socio-politico. In questo senso, appare innegabile che il fine ultimo di ogni politica linguistica promossa dalle classi egemoniche al potere (come appunto nel caso del fascismo) sia quello di creare, tramite l'imposizione di interventi linguisticamente coercitivi, le premesse per una consapevole strategia a tutto campo di manipolazione dell'individuo (e quindi della comunità parlante) a livello anche socio-pragmatico, attuata mediante una consapevole opera di manipolazione della lingua.

Inquadrando la politica linguistica adottata dal fascismo nell'ambito del processo della questione della lingua in Italia, ci pare che l'elemento più significativo di tale fase storica sia rintracciabile, al di là dei limiti del programma di restaurazione delle tendenze puristiche, nel nesso di relazione dialettico e sincronico con un complesso intreccio di implicazioni socio-pragmatiche. Implicazioni, queste, che offrono lo spunto per una serie di riflessioni di natura sociolinguistica su alcuni concetti chiave, suscettibili di un necessario approfondimento, quali pianificazione linguistica, intenti normativi, cultivation approach, policy approach, ecc.

Di particolare rilievo ci pare soprattutto la circostanza che la questione della lingua, in una fase storica involutiva, che coincide con l'attuazione di un rigoroso purismo di stato, espressione di un'ideologia nazionalistica, tocchi il suo punto di massima, gettando nello stesso tempo le premesse per un superamento delle tradizionali caratteristiche, marcatamente teoriche e letterarie, per assumere spiccate connotazioni sociologiche.

Nella fase storica del «purismo di stato », oggetto delle nostre riflessioni, ci pare pertanto ravvisabile uno spartiacque significativo verso il superamento della tradizionale antinomia «norma - uso », che aveva accompagnato costantemente nei secoli la questione della lingua in Italia. Il significato della svolta va ricercato secondo noi nella sempre più diffusa percezione dell'imprescindibile nesso di interrelazione fra dinamica linguistica e fattori sociologici (migrazioni interne, industrializzazione, urbanesimo, mass-media alfabetici e non alfabetici).

Un nesso interagente questo, che, a partire dagli anni Sessanta, con l'avvento della sociolinguistica, diverrà, in particolare in Italia, un fondamentale elemento di stimolo e di riferimento per le ricerche della sociolinguistica. Quest'ultima, rovesciando il tradizionale ordine gerarchico fra linguistica esterna e quella interna a vantaggio della prima, assegnerà una forte rilevanza pragmatica ai fattori di varietà e di stratificazione della lingua, sia orizzontale che verticale (bilinguismo, diglossia, varietà linguistiche). Fattori questi che erano stati già al centro delle riflessioni degli esponenti del neopurismo di stato, ma che dopo l'avvento della sociolinguistica verranno dialetticamente correlati al comportamento linguistico non più di una comunità di parlanti intesa come astrattamente unitaria o idealmente omogenea, ma composta da categorie di utenti differenziate, storicamente e concretamente caratterizzate. 


\section{BIBLIOGRAFIA}

BARTOLI Matteo (1930), I dialetti dell'Italia settentrionale, nota estratta dal vol. Piemonte. Guida d'Italia del T.C.I., Milano. In: De MAURo TUlLIO (1970).

BERRUTO Gaetano (1974), La sociolinguistica, Bologna, Zanichelli.

BOLELLI Tristano (1971), Linguistica generale, strutturalismo, Linguistica storica. Testi, note introduttive, indici, Pisa, Nistri-Lischi.

DARDANO Maurizio (1978), G.I.Ascoli e la questione della lingua, Roma, Christen DE MAURO Tullio (1970), Storia linguistica dell'Italia unita, Bari, Laterza.

Devoto Giacomo (1974), Il linguaggio d'Italia, Milano, Rizzoli.

FishMAN Joshua A. (1972), La sociologia del linguaggio (trad. it. di M. Gnerre, con un saggio introduttivo di A.M.Mioni, Roma, Officina, 1975).

KLEIN Gabriella (1986), La politica linguistica del Fascismo, Bologna, il Mulino.

LEPSCHY Giulio Carlo (a cura di, 1994), Storia della linguistica, vol.III, Bologna, Il Mulino.

LOMBARDO Radice Giuseppe (1925), Il dialetto nella scuola. In: Accanto ai maestri, Torino.

Menarini Arturo (1941), A proposito di, LN III. In : De MAURo TUlLIO (1970).

MIGLIORINI Bruno (1941), La lingua nazionale, Firenze, Le Monnier.

PASOLINI Pier Paolo (1969), Nuove questioni linguistiche, in «La nuova questione della lingua $»$. Saggi raccolti da O.Parlangeli, Bari.

RAFFAELLI Sergio (1983), Le parole proibite, Bologna, il Mulino.

SAUSSURE Ferdinand de (1916), Cours de linguistique générale, a cura di Ch.Bally e A.Sechehaye, Lausanne/ Paris, Payot (traduzione italiana con introduzione e commento di T. De Mauro, Corso di linguistica generale, Bari, Laterza, 1967).

Vitale Maurizio (1978), La questione della lingua, Palermo, Palumbo.

\section{ABSTRACT}

We note during fascism in Italy one important historic phase of the linguistic issue. This time it is linked with the revival of puristic trends, connected with the introduction of prescriptive language policy It was puristically oriented (the socalled «state purism») against dialects and against the national language minorities in Italy, but particularly against the presence of foreign-language elements. In this regard, we put stress particularly on the fact that those who in a different measure took part in these puristic regulatory interventions, were the most prominent linguists of those days, e.g. B. Migliorini, A. Schiaffini, G. Bertoni, etc. We pay special attention to the analysis of the core issue of the fascism language policy outlining the necessary theoretical picture, linked with the language policy and language planning. Within the inseparable integrity between the dynamics of the development of the society and the language dynamics, we emphasize to important interactions and the sociological factor functioning like language. 